disease of the joints. Whilst the autoimmune element continues to be studied intensely, it is evident that the innate inflammatory response propagates the disease. Fibroblast-like synoviocytes (FLS) are the most abundant stromal cell in the synovium. FLS from RA joints are markedly different from their healthy counterparts in their inflammatory characteristics.

RA FLS exhibit a form of inflammatory memory named priming. Prolonged exposure to TNF $\alpha$ induces an augmented chemokine response to challenges with interferons [1]. Fibroblast priming leads to significantly augmented IL6, CCL5 and CXCL10 responses to a second challenge by $\mathrm{TNF} \alpha$, with a coincident prolonged $\mathrm{NF}_{K} \mathrm{~B}$ nuclear localization (manuscript in press).

Objectives: We wished to assess whether fibroblasts from sites other than the synovial joint would also exhibit this primed response to second challenge.

Methods: Fibroblasts from the joint, skin, lung, tonsil, bone marrow and gum were stimulated with $10 \mathrm{ng} / \mathrm{ml} \mathrm{TNF} \alpha$ for $24 \mathrm{~h}$, before the conditioned medium was removed and the cells were washed free of stimulus. Cells were rested for $24 \mathrm{~h}$ before once again being washed and stimulated with $10 \mathrm{ng} / \mathrm{ml} \mathrm{TNF} \alpha$ for a further $24 \mathrm{~h}$. The conditioned medium was removed and secreted mediators compared between first and second response to stimulus. Transcription and intracellular signalling at time points within each challenge were also determined.

Results: Synovial, lung and tonsil fibroblasts augmented IL6 secretion in response to the second TNF $\alpha$ challenge. RA bone marrow-derived fibroblasts varied in their exhibition of priming. Skin fibroblasts from patients undergoing cosmetic surgery and a gingival sample from non-chronic inflammation displayed no evidence of IL6 priming.

Fibroblasts from the skin of psoriasis (Ps) patients mounted a primed response that was significantly augmented compared to their first response to TNF $\alpha$, and significantly higher than the second response of healthy skin. This augmented response matches that of FLS, as IL6 but not IL8 was increased. This pattern was also matched by gum fibroblasts from periodontitis.

Mechanistically the Ps skin fibroblasts match FLS, in that NF $F B$ nuclear localization is prolonged in the second challenge compared to the first.

Conclusions: We have shown that inflammatory memory in the form of IL6 priming occurs in fibroblasts from a variety of anatomical sites with diverse functions. Its prevalence implies a shared phenomenon, but the variation between sites suggests a specific role required in some tissues and not others.

The finding that Ps skin fibroblasts acquire this memory response may point towards a pro inflammatory mechanism that contributes to psoriatic diseases, including psoriatic arthritis. Our data may help to explain why an estimated $30 \%$ of psoriasis patients go on to develop psoriatic arthritis [2]

References:

[1] Sohn, C., et al., Prolonged TNFaprimes fibroblast-like synoviocytes in a gene-specific manner by altering chromatin. Arthritis Rheumatol, 2014.

[2] Mease, P.J., et al., Prevalence of rheumatologist-diagnosed psoriatic arthritis in patients with psoriasis in European/North American dermatology clinics. $J$ Am Acad Dermatol, 2013. 69(5): p. 729-35

Disclosure of Interest: None declared

DOI: 10.1136/annrheumdis-2017-eular.5179

\section{OP0179 BRAFV600E PROMOTES MYELOID SKEWING IN MULTISYSTEMIC LANGERHANS CELL HISTIOCYTOSIS HUMANIZED MOUSE MODEL}

$\underline{R . ~ B i a v a s c o ~}^{1}$, M. Norelli ${ }^{2}$, B. Camisa ${ }^{2}$, D. Cesana ${ }^{1}$, P. Gallina ${ }^{1}$, B. Gentner ${ }^{1}$ M. Ponzoni ${ }^{3}$, L. Dagna ${ }^{4}$, A. Bondanza ${ }^{2}$, E. Montini ${ }^{1}$. ${ }^{1}$ Sr-Tiget; ${ }^{2}$ Innovative immunotherapy; ${ }^{3}$ Pathology Unit; ${ }^{4}$ Clinical immunology and rheumatology, San Raffaele Scientific Institute, Milan, Italy

Background: Multisystemic Langerhans cell histiocytosis ( $\mathrm{mLCH}$ ) is an aggressive disease characterized by the accumulation of mononuclear phagocytes with immunohistochemical features of dendritic cell (histiocytes) ${ }^{1}$. Histiocytes infiltrate mostly the skin, bone marrow (BM), lung and spleen, and produce high levels of proinflammatory cyto/chemokines, leading to organ dysfunction ${ }^{2,3}$. In patients, around $10 \%$ of cells in lesions carries an oncogenic mutation in the MAPK pathway, mostly BRAF ${ }^{\mathrm{V} 600 \mathrm{E}}$ (70\% of cases). BRAF ${ }^{\mathrm{V} 600 \mathrm{E}}$ can be detected also in monocytes and BM progenitors (HSPC) from these patients, whereas only a fraction of them carries a mutation in B cells and none in T cells ${ }^{4}$.

Objectives: To study the role of $\mathrm{BRAF}^{\mathrm{V} 600 \mathrm{E}}$ in the pathogenesis of $\mathrm{mLCH}$, we set up a humanized mouse model of $\mathrm{mLCH}$ based on the transplantation into immunodeficient mice (NSG) of human HSPC expressing BRAFV600E

Methods: We isolated HSPC from human cord blood and transduced them at two different levels (50\% and $20 \%$ ) with lentiviral vectors that ubiquitously express BRAF $^{\mathrm{V} 600 \mathrm{E}}$, BRAFWT ${ }^{\mathrm{WT}}$ or GFP.

Results: All BRAF ${ }^{\mathrm{V} 600 \mathrm{E}}$ mice manifested severe weight loss within 7 weeks with a median of 3 and 5 weeks for $50 \%$ and $20 \%$ transduction groups, respectively. Mice showed dysplastic bone marrow (BM) with infiltration of histiocytes; lesions were present also in lungs, kidneys, CNS, spleen and liver. Immunophenotype of infiltrating histiocytes closely resembles $\mathrm{mLCH}$, staining positive for CD14, CD68, S-100 and langerin. None of the control mice lost weight nor displayed organ alteration. Flow cytometry analyses of BM showed 5- to 7-fold reduction in engraftment of human cells in BRAF ${ }^{\mathrm{V} 600 E}$ vs GFP groups $(p<0,001)$. Percentage of myeloid cells increased by 3 - to 4 -fold in BRAFV600E vS GFP groups $(p<0,001)$. On the contrary, percentage of $B$ cells was reduced by 3 - to 6,5 -fold in BRAF $^{\mathrm{V} 600 \mathrm{E}}$ vs GFP groups $(\mathrm{p}<0,001)$. Moreover, there was no difference in the percentage of GFP-positive cells between myeloid cells, whole BM and in vitro sample, suggesting a non-cell autonomous mechanism underlying this myeloid phenotype.

Conclusions: In summary, we generated for the first time a human xenogeneic transplantation mouse model of $\mathrm{mLCH}$, showing that BRAF ${ }^{\mathrm{V} 600 \mathrm{E}}$ in human HSPC promotes myeloid skewing rather than proliferation.

\section{References:}

[1] Wilejto et al. Curr Opin Rheumatol 2012. DOI: 10.1097/BOR. 0b013e32834db53e.

[2] Donadieu et al. Histiocytic Disorders of Children and Adults 2005.

[3] Kannourakis et al. Br J Cancer 1994, Sep;23:S37-40.

[4] Berres et al. J Exp Med 2014, DOI: 10.1084/jem.20130977.

Disclosure of Interest: None declared

DOI: 10.1136/annrheumdis-2017-eular.1840

\section{THURSDAY, 15 JUNE 2017}

\section{Joint health \& joint damage: a tale of three tissues _}

\section{OP0180 PHARMACOLOGICAL CHARACTERIZATION OF THE ADAMTS-5 INHIBITOR GLPG1972: AN ORAL ANTI-CATABOLIC AGENT FOR THE TREATMENT OF OSTEOARTHRITIS}

P. Clement-Lacroix ${ }^{1}$, C. Little $^{2}$, R. Blanqué ${ }^{1}$, S. Meurisse ${ }^{1}$, P. Mollat $^{1}$ F. Brebion ${ }^{1}$, R. Gosmini ${ }^{1}$, F. De Ceuninck ${ }^{3}$, I. Botez ${ }^{3}$, L. Lepescheux ${ }^{1}$, E. van der Aar ${ }^{4}$, T. Christophe ${ }^{4}$, N. Vandervoort ${ }^{4}$, C. Cottereaux ${ }^{1}$, D. Comas ${ }^{1}$, P. Deprez ${ }^{1}$, D. Amantini ${ }^{1}$. ${ }^{1}$ Galapago SASU, Romainville, France; ${ }^{2}$ Raymond Purves Bone and Joint Research Laboratories, University of Sydney, Kolling Institute, Northern Sydney Local Health District, Sydney, Australia; ${ }^{3}$ Institut de Recherches Servier, Suresnes, France; ${ }^{4}$ Galapagos NV, Mechelen, Belgium

Background: Degradation of articular cartilage and alterations of the underlying subchondral bone are hallmarks of osteoarthritis $(O A)^{1}$. A disintegrin and metalloproteinase with thrombospondin motifs-5 (ADAMTS-5) is a key aggrecancleaving enzyme involved in this pathogenic process from the earliest stages of cartilage degradation ${ }^{2}$ and as such, is an attractive drug target for the development of a disease-modifying OA drug (DMOAD) ${ }^{3}$.

Objectives: In this report we describe the in vitro and in vivo characterization of the small molecule GLPG1972, an inhibitor of ADAMTS-5. GLPG1972 anticatabolic activity was evaluated in murine and human cartilage explants and DMOAD activity was investigated in the destabilization of the medial meniscus (DMM) mouse model $^{4}$.

Methods: The ADAMTS-5 biochemical assay is based on the cleavage of a fluorescent substrate by recombinant ADAMTS-5. Mouse femoral head cartilage explants were stimulated by interleukin-1a (IL-1a) for 3 days and GAG release quantified $^{2 b}$. Human articular cartilage explants were stimulated with IL-1 $\beta$ for 12 or 19 days and the NITEGE epitope quantified using the AGNx1 assay. Unilateral $\mathrm{OA}$ was induced in C57BL6 mice by $\mathrm{DMM}^{4}$. Mice were treated with vehicle or GLPG1972 at 30, 60 or $120 \mathrm{mg} / \mathrm{kg}$, b.i.d. for 8 weeks. Medial femorotibial joint sections were scored by an evaluator blinded to treatment.

Results: GLPG1972 showed potent inhibition of human ADAMTS-5 $\left(\mathrm{IC}_{50}=20 \mathrm{nM}\right)$. Inhibition of ADAMTS-4 was moderate $\left(\mathrm{IC}_{50}=57 \mathrm{nM}\right)$, and selectivity over 100 -fold was observed against a large panel of zinc metalloproteinases. GLPG1972 displayed potent anti-catabolic activity in cartilage explants, with $\mathrm{IC}_{50}$ values being $2 \mu \mathrm{M}$ and $<1 \mu \mathrm{M}$ in mouse and human, respectively. In the DMM mouse model, GLPG1972 demonstrated DMOAD activity, as shown by significant reduction of femorotibial cartilage proteoglycan loss and cartilage damage score, as well as significant impact on subchondral bone sclerosis.

Conclusions: GLPG1972 is an orally bioavailable, potent and selective ADAMTS 5 inhibitor showing significant anti-catabolic activity in cartilage explants. In the DMM model, treatment with GLPG1972 resulted in significant protective effects on both cartilage and subchondral bone pathology. Taken together these results provide support to progress GLPG1972 into the clinic as an oral treatment for OA. References:

[1] Hunter, D.J., et al. Curr. Opin. Rheumatol. 2009, 21,110-117.

[2] a) Glasson, S.S., et al Nature. 2005, Vol 434: 644-8; b) Stanton, H., et al. Nature. 2005, Vol 434: 648-5; c) Little, C.B., et al. Journal of Clinical Investigation. 2007, Vol 117(6):1627-36.

[3] Larkin, J. et al. Osteoarthritis Cartilage. 2015, 23 (8): 1254-1266.

[4] Little, C.B. and Hunter, D.J. Nat. Rev. Rheumatol. 2013, 9(8):485-497.

Disclosure of Interest: None declared

DOI: 10.1136/annrheumdis-2017-eular.3775

\section{OP0181 DELETION OF THE PROSTAGLANDIN D2 RECEPTOR DP1 EXACERBATES AGING-ASSOCIATED AND INSTABILITY-INDUCED OSTEOARTHRITIS}

Y. Ouhaddi, L. Habouri, J.-P. Pelletier, J. Martel-Pelletier, M. Benderdour, H. Fahmi. Medicine, CRCHUM, Montreal, Canada

Background: The $D$ prostanoid receptor 1 (DP1), a receptor for prostaglandin $D_{2}$ $\left(\mathrm{PGD}_{2}\right)$ plays important roles in inflammation and cartilage metabolism. However, its role in the pathogenesis of osteoarthritis (OA) remains unknown. 